\title{
URBAN AREA EXTRACTION IN SAR DATA
}

\author{
H. Aghababaee*, S. Niazmardi , j.Amini
}

Dept. of Geomatics Engineering, College of Engineering, University of Tehran, Tehran, Iran

KEY WORDS: SAR data, Urban Area Extraction, Feature Selection, Classification

\begin{abstract}
:
In this paper, the performance of different texture measures for detection of urban areas from SAR data is evaluated. The used texture measures are categorized into two groups, the first group include the SAR specific textures and the second one considers the general texture measures. ffmax is selected from the first category and LISA, SRPD, Wavelet measures and fractal dimensions are used as general texture measures. For a better discrimination, all texture measures are calculated and a PCA rotation is applied to them and the first PC is multiplied by the urban inhomogeneity parameter and the obtained image is segmented. The obtained results of this procedure comparing with the K-Means clustering algorithm show the better performance of this algorithm for urban area detection.
\end{abstract}

\section{INTRODUCTION}

Today, managing the rapid growth of urbanization and population of the cities require the up-to-date information about urban area. Urban managers and decision makers use this information from any possible data sources. One the best source of information for urban area is remote sensing. Remotely sensed data due to their vast coverage, frequent observation and fair prices are widely used in urban analysis. Nevertheless, the usage of optical remote sensing data in VIS/NIR portion of the electromagnetic spectrum is usually limited by lots of factors such as cloud contamination, atmospheric effect, and presence of difference materials in complex urban areas. Consequently, they cannot have an acceptable performance. On the other hand, radar sensors because of being independent of atmospheric and weather conditions and not being affected by cloud have gained more attentions. These factors in addition to the high resolution SAR sensors make these sensors the best choice for the urban analysis.

Urban objects, depending on their geometry and roughness as well as their effects on the local statistical characteristics of images, could be classified into three sets: smooth surfaces, rough surfaces, and conductor surfaces. The backscatter law of smooth surfaces are close to Snell-Descartes' ones. Rough surfaces represent irregularities on the scale of wavelength, and conductor surfaces are mainly metallic ones, which are never plan and act as periodic lattices. The composition of the three previous effects determines the characteristics of the global backscattering law: 1) there are more dark pixels, because of the Snell Descartes responses in an unfavourable configuration; 2) there are more bright pixels, because of the Snell-Descartes responses in a favourable configuration; and 3) the intermediate values around the mean caused by speckle are not so much modified (He et al., 2006).

Considering these properties, the algorithms proposed for urban area detection can be categorized to three categories.

The first category consists of statistically based methods. These methods threat each radar backscatterer as a random variable and try to detect the urban area by fitting some probability density function to back scattering coefficients (Gamba et al., 2005; Tison et al., 2004; Yang et al., 2006). The second category includes texture based analysis. As their name implies these methods try to extract some texture measure from VHR SAR data to discriminate between urban and non-urban areas (Dekker, 2003; Dell'Acqua and Gamba, 2001; He et al., 2006). The last category uses the data fusion techniques to fuse the SAR data with other kind of data for better classification of urban areas. The SAR data is fused with both LIDAR and optic data in the literature for this purpose (Gamba et al., 2005; Gomez-Chova et al., 2006; Soergel et al., 2005).

The purpose of this paper is to study the methods of second category. For these purpose defend texture measure are calculated using SAR data and their ability for detection of urban area is evaluated by classification.

The rest of this paper is outlined as follows. In the second section, the used texture measures are presented. Dataset and implementation of the algorithms are discussed in section three; finally we draw our conclusion in section four.

\section{TEXTURE MEASURES}

The used texture measures in this study are categorized into two categories. The first category includes SAR specific textures, the second category are general textures that can be calculated for every image data. In the following subsections these two categories are detailed.

\subsection{SAR specific textures}

\subsubsection{FFMAX}

This texture measure, is proposed as an algorithm for detection of urban area from SPOT imagery and then it was used for detection of urban area from the SAR data (Gouinaud et al., 1996). This method that is mostly a descriptor for urban areas, assume that the amplitude image, takes value from [0 $g$-1] where $g$ is the maximum gray level of SAR amplitude image. Then the algorithm uses threshold $s, s \in[0, g-1]$ to cut the local histogram (on $60 \times 60$ window). Then the probability of the higher part $\left(\sum=P(A>s)\right)$ is calculated and compared to the highest statistical frequency of the lower part $\left(f_{\max }\right)$. When

\footnotetext{
* Corresponding author.
} 
$\sum>f_{\max }$ the central pixel receives the value of $s$ as its current value or the value should be decreased. This process iterates for all pixels (He et al., 2006).

Thus texture measure, not only extract urban measures but also measure urban agglomeration density, and as mentioned provide a low level descriptor for urban areas with no precise border (He et al., 2006).

\subsection{General Textures}

2.2.1 Local Indicator of Spatial Association (LISA)

LISA indices are local indicators of spatial autocorrelation, which are widely used in GIS studies. In this study two LISA indices are used namely Maran's I and Geary's C. Moran's I index evaluate the similarity between the neighbours of a pixel by comparing its value with average local value. Thus, it describes the local homogeneity using the following equation.

$$
I_{i}=\frac{x_{i}-\bar{x}}{\sum_{i} \frac{\left(x_{i}-\bar{x}\right)^{2}}{n}} \sum_{j} w_{i j}\left(x_{i}-\bar{x}\right)
$$

Where, $x$ and $w$ denote the value of amplitude image and the weight matrix, which is used to define the neighbourhood. The I index is within the range of [-1 1$]$.

Geary's C index identify area of high contrast, providing a measure of local dissimilarity, according to

$$
c_{i}=\frac{1}{\sum_{i} \frac{\left(x_{i}-\bar{x}\right)^{2}}{n}} \sum_{j} w_{i j}\left(x_{i}-x_{j}\right)^{2}
$$

The value of this index is within the range of $[1,2]$ and the upper limit refers to strong negative spatial correlation, while the lower one corresponds to uncorrelated data (Gamba et al., 2011).

\subsubsection{Square Root Pair Difference (SRPD)}

SRPD is a semi-variogram texture measure, which is calculated using the following equation:

$$
S R P D=\frac{1}{N_{h}} \sum\left[\operatorname{abs}\left(x_{i}-x_{i+h}\right)\right]^{1 / 2}
$$

In this equation, $h$ is the lag distance in a predefined direction and $N_{h}$ is the number of considered neighbour pixels (Dong et al., 2011).

\subsubsection{Wavelet texture Measure}

The wavelet transform is widely used as texture measure for SAR and optic remotely-sensed data. It is known that the Daubechies wavelet with four coefficients has the highest classification accuracy for SAR data (Dekker, 2003).

\subsubsection{Fractal Dimension}

Fractal dimension can estimate the degree of self similarity of the image. The fractal dimensions have been used successfully in texture analysis and segmentation of images. There are many methods proposed to estimate the fractal dimension of the image that usually has similar values. In this study the box count method is used (Dekker, 2003).

\section{EXPRIMENTAL RESULTS}

The implemented framework is shown in Fig.1. For our experiments, we used SAR data from the urban area in Japan.
The data comes from the TerraSAR-X, X-band SAR sensor and is illustrated in Fig.2.

The mentioned textures are calculated for two portions of the used data. Due to the nature of textures, they highlight the urban areas in different ways for some of them such as FFMAX and fractal dimension the urban areas have higher value and for some of them they have lower values. To take advantage of all textures, a PCA transform is applied to them. By applying PCA transform, it is possible to show all features within the same frame work. After applying PCA, its first component is multiplied by a man-made structure highlighting parameter. The reason of using the first PC is that it is well-known that this PC has the highest level of spatial information in comparison to the rest of them. Finally, by segmenting this image, the urban areas are extracted.

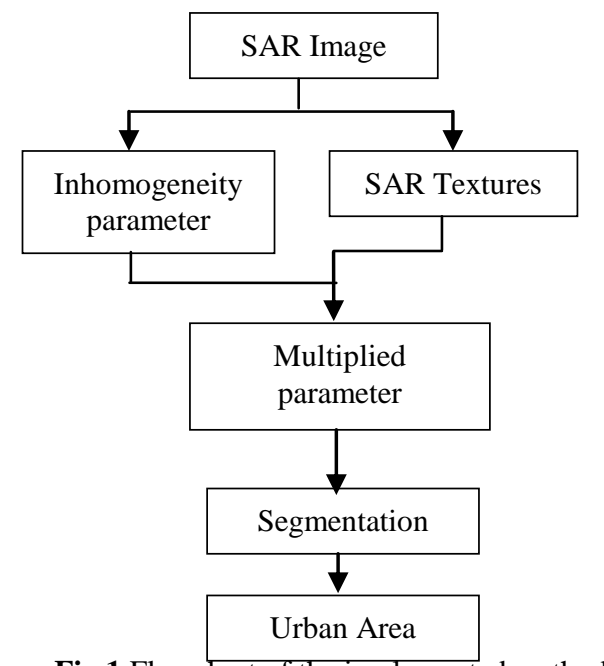

Fig.1 Flowchart of the implemented method

Since the man-made structures are typically characterised by sudden changes in brightness at edges and in layover, shadow, etc, an easy way to describe the texture inhomogeneity is the filtering coefficient of the LEE speckle filter (Lee, 1980). In the LEE filter, de-speckled estimates of the pixel intensity $x$ are obtained from the original intensity $x$ as follows.

$$
x^{\prime}=\bar{x}+k(x-\bar{x})
$$

In this equation, $\bar{x}$ denoting the local mean of the measured amplitudes $x$. The factor $k$ can be expressed as follow equation.

$$
k=\frac{\operatorname{var}(x)-\bar{x}^{2} \sigma_{n}^{2}}{\operatorname{var}(x)\left(1+\sigma_{n}^{2}\right)}
$$

The $k$ factor becomes 0 over homogenous areas and tends to 1 over point targets and edges. $\sigma_{n}^{2}$ describes the a-priori speckle variance and is equal to 1 over the number of looks. However, since the correct estimation of the local variance $\operatorname{var}(x)$ and of the local mean $\bar{x}$ requires the use of large windows, the computation of $k$ entails a significant degradation of resolution. The estimation result can significantly be improved by multiplying $k$ by the image amplitude $(\varphi=k x)$ (Reigber et al., 2007). $\varphi$ becomes very large over point targets, where both the texture inhomogeneity and the image amplitude are large, and is well suited for discrimination of man-made structures. The computed inhomogeneity parameter $(\varphi)$ and the first component of PCA are multiplied and the multiplied image is classified by the implemented segmentation method. 
Fig. 2 depicts, the calculated inhomogeneity parameter $(\varphi)$ from the amplitude images of the TerraSAR-X data sets. In this paper in order to segments the multiplied image to the urban and un-urban area, a binary decision is considered. The binary decision is made by applying a threshold on the multiplied image. The threshold is computed using the well-known Otsu method (Otsu, 1979).

Fig. 3 shows the results of the urban extraction by the implemented method.

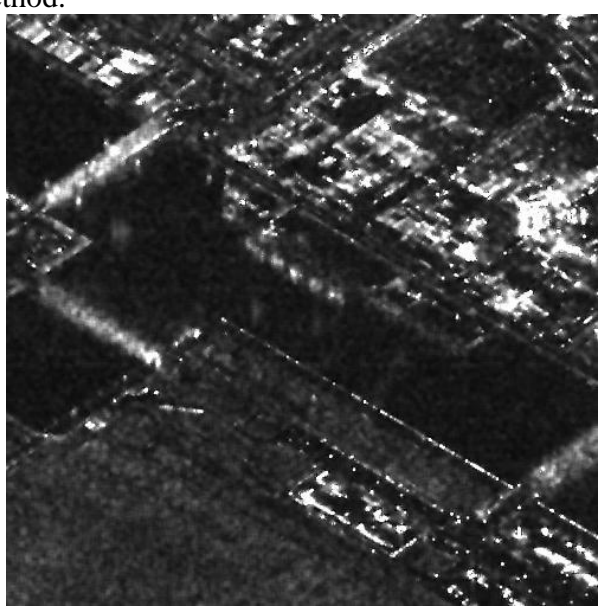

(a) First data set

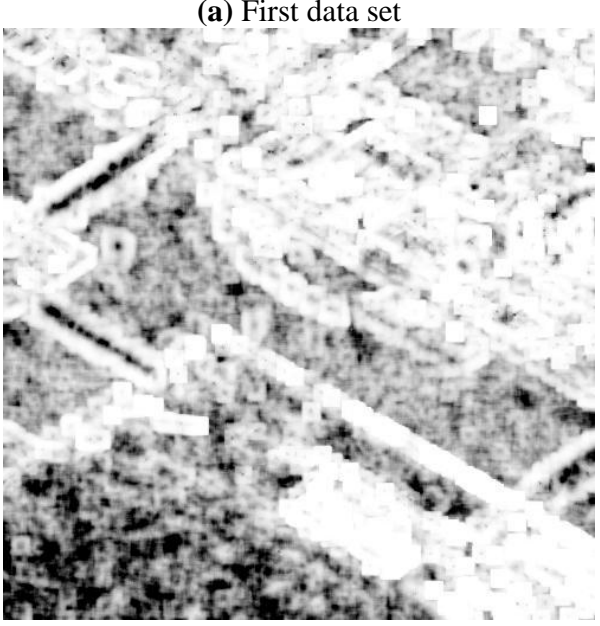

(c) inhomogeneity parameter of the first data set

Fig.2 Samples of TerraSAR-X images for the test urban area and their inhomogeneity parameters

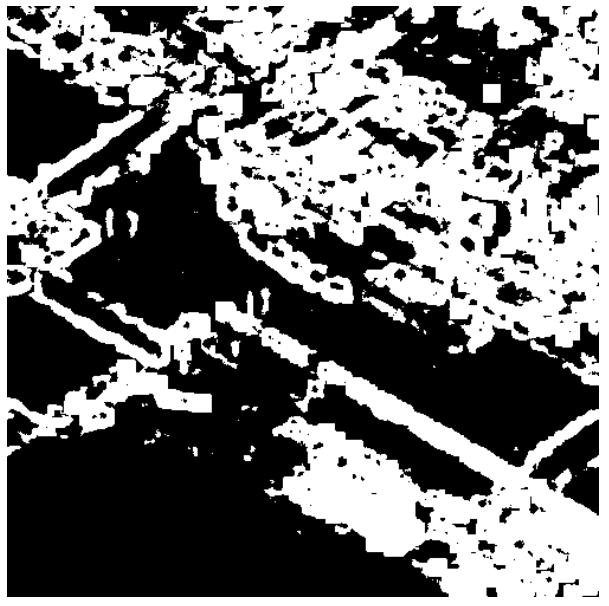

(a) urban area in data set 1
For comparison the results of the implemented method are compared with the results of clustering the textures using kmeans algorithms and the results are shown in fig 4 .

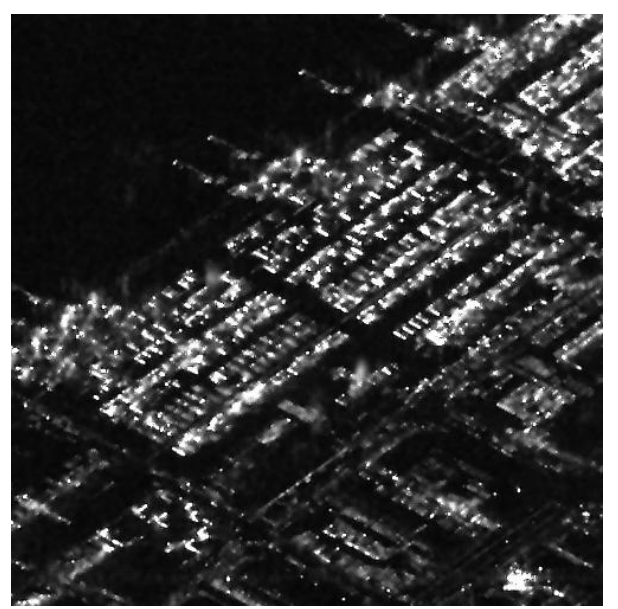

(b) Second data set

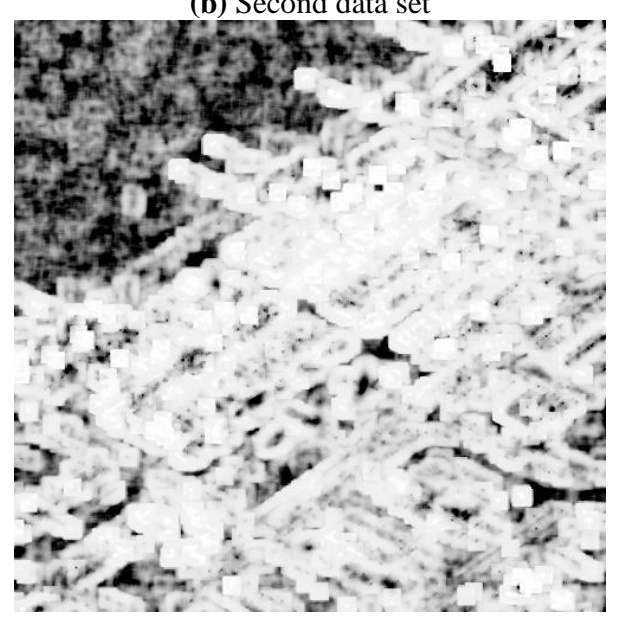

(d) inhomogeneity parameters of the second data set

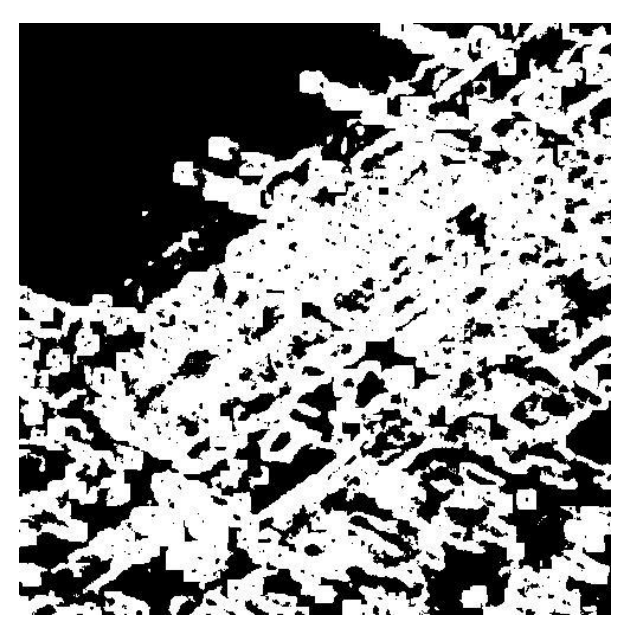

(b) urban area in data set 2

Fig.3 Urban extraction maps of the study areas using the proposed method. 


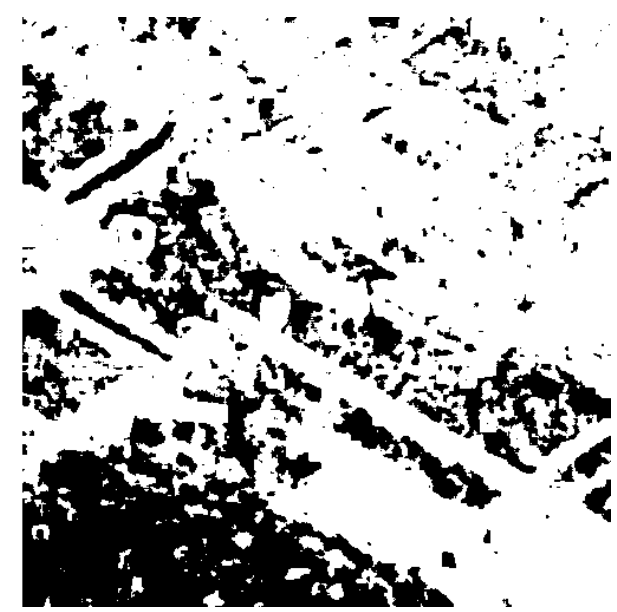

(a) urban area in data set 1

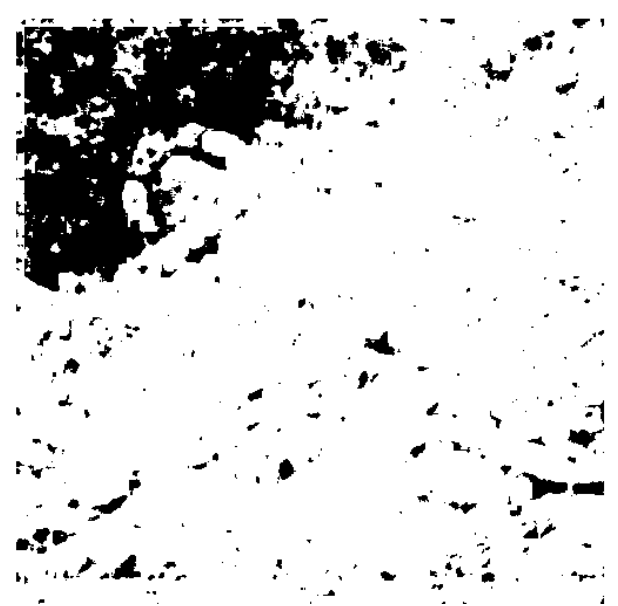

(b) urban area in data set 2

Fig.4 Urban extraction maps of the study areas using the K-means method.

Comparing fig. 3 and fig. 4 , it is obvious that the proposed method has much better performance. In the results of K-means algorithms the streets at the urban area are mostly classified as urban area. More over it can be seen that the proposed method have classified both the urban and non-urban area more accurate.

It should be noted that a Lee filter is chosen to reduce speckle effects with a window size of $5 \times 5$. According to Fig. 3, qualitative analysis shows the efficiency of the implemented method in different test areas. Man-made and urban areas are properly distinct from other types of land covers in the images. The efficiency of the methods is related to the textures which are extracted from the original SAR image as well as the used inhomogeneity parameter. By analysis of the inhomogeneity parameters in Fig. 2, one can conclude that, $\varphi$ in the areas without buildings, is very close to zero and both its mean and variance are small. One important point in the implemented framework is the widow size which is used in texture and inhomogeneity parameter computations. In other word, there is a trade-off in choosing the window size for the texture analysis. Indeed, as the window size increases, the texture feature is better estimated in terms of statistics robustness, but the uncertainty area between two different textures also gets larger, and edges are not accurately localized. A window size of $15 \times$ 15 pixels has proved to be a good compromise for images with spatial resolutions ranging between 5 and $20 \mathrm{~m}$ [17]. However using of the fuzzy logic seems to be interesting algorithm in computing the window size.

\section{CONCLUSION}

In this paper, a technique for the detection of the man-made structures using very high resolution SAR images is proposed. Basically, the procedure starts with the calculation of different textures from SAR data. The textures are extracted from the amplitude images. Since not all these textures are informative, a feature reduction step is used. Among different feature extraction methods, in this study the well-known principal component analysis (PCA) has been adapted. Then a simple and powerful estimator called inhomogeneity parameter for the urban structures highlighting is computed and multiplied by the first component of PCA. Finally the urban areas are extracted from the multiplied image using a binary decision method. Results on the very high resolution (VHR) TerraSAR-X images show that the method has high efficiency for unsupervised man- made structure extraction. However, the framework needs to be applied to an enormous SAR image database and also to the different SAR sensors image.

\section{Reference}

Dekker, R.J., 2003. Texture analysis and classification of ERS SAR images for map updating of urban areas in the Netherlands. Geoscience and Remote Sensing, IEEE Transactions on 41, 1950-1958.

Dell'Acqua, F., Gamba, P., 2001. Detection of urban structures in SAR images by robust fuzzy clustering algorithms: the example of street tracking. Geoscience and Remote Sensing, IEEE Transactions on 39, 2287-2297.

Dong, Y., Chen, H., Yu, D., Pan, Y., Zhang, J., 2011. Building extraction from high resolution SAR imagery in urban areas. Geo-spatial Information Science 14, 164-168.

Gamba, P., Aldrighi, M., Stasolla, M., 2011. Robust extraction of urban area extents in HR and VHR SAR Images. Selected Topics in Applied Earth Observations and Remote Sensing, IEEE Journal of 4, 27-34.

Gamba, P., Dell'Acqua, F., Dasarathy, B.V., 2005. Urban remote sensing using multiple data sets: Past, present, and future. Information Fusion 6, 319-326.

Gomez-Chova, L., Fernández-Prieto, D., Calpe, J., Soria, E., Vila, J., Camps-Valls, G., 2006. Urban monitoring using multitemporal SAR and multi-spectral data. Pattern recognition letters 27, 234-243.

Gouinaud, C., Tupin, F., Maitre, H., 1996. Potential and use of radar images for characterization and detection of urban areas, Geoscience and Remote Sensing Symposium, 1996. IGARSS'96.'Remote Sensing for a Sustainable Future.', International. IEEE, pp. 474-476.

He, C., Xia, G., Sun, H., 2006. An adaptive and iterative method of urban area extraction from SAR images. Geoscience and Remote Sensing Letters, IEEE 3, 504-507. 
Reigber, A., Jager, M., He, W., Ferro-Famil, L., Hellwich, O., 2007. Detection and classification of urban structures based on high-resolution SAR imagery, Urban Remote Sensing Joint Event, 2007. IEEE, pp. 1-6.

Soergel, U., Schulz, K., Thoennessen, U., Stilla, U., 2005. Integration of 3D data in SAR mission planning and image interpretation in urban areas. Information Fusion 6, 301-310.

Tison, C., Nicolas, J.M., Tupin, F., Maître, H., 2004. A new statistical model for Markovian classification of urban areas in high-resolution SAR images. Geoscience and Remote Sensing, IEEE Transactions on 42, 2046-2057.

Yang, Y., Sun, H., Cao, Y., 2006. Unsupervised urban area extraction from SAR imagery using GMRF. Pattern Recognition and Image Analysis 16, 116-119.

Lee,S., "Digital image enhancements and noise filtering by use of local statistics," IEEE Transactions on Pattern Analysis and MachineIntelligence, vol. PAMI-2, pp. 165-168, 1980

OTSU, N., 1979: A Threshold Selection Methodfrom GrayLevel Histograms. IEEE Transactions on Systems, Man and Cybernetics 9 (1): 62-66. 